\title{
THE TEVATRON TRIPLER - MAGNET DESIGN AND LATTICE ISSUES*
}

\author{
A. McInturff ${ }^{\dagger}$, P. McIntyre ${ }^{\dagger \dagger}$, A. Sattarov, Texas A\&M University, College Station, TX 77840 USA
}

\begin{abstract}
Recent advances in superconductor properties and superconducting magnet technology have made it possible to build cost-effective, high-performance dipoles with a field strength of 13 Tesla - 3 times that of the Fermilab Tevatron. Such magnets could be used to upgrade Fermilab's collider in its existing tunnel to a collision energy $\sqrt{s}_{\mathrm{s}}$ $=6 \mathrm{TeV}$ and luminosity $\mathcal{L}=10^{33} \mathrm{~cm}^{-2} \mathrm{sec}^{-1}$. A design is presented for a 13 Tesla $\mathrm{Nb}_{3} \mathrm{Sn}$ dipole suitable for the separated orbits of protons and antiprotons. The requirements for beam separation, low- $\beta$ insertions, and abort are discussed. Compatibility of the higher collision energy and luminosity for study using the detectors CDF and D0 is discussed.
\end{abstract}

\section{THE TEVATRON TRIPLER}

The Fermilab Tevatron [1] was the world's first highenergy superconducting ring. It has supported a rich program of physics with proton-antiproton colliding beams for the past 20 years. Recent upgrades of the main injector, antiproton source, and recycler ring will dramatically increase the antiproton accumulation rate and the luminosity of collisions in the two experiments.

A recent paper [2] suggested that the Tevatron could be upgraded by replacing its single ring of 4.2 Tesla magnets with a single ring of 12 Tesla magnets, using the $\mathrm{Nb}_{3} \mathrm{Sn}$ magnet technology that is being developed at LBL [3], Fermilab [4], and Texas A\&M [5]. The result would be a $\bar{p} p$ collider with energy $\sqrt{\mathrm{s}}_{\mathrm{s}}=6 \mathrm{TeV}$ and luminosity $\mathcal{L}=$ $10^{33} \mathrm{~cm}^{-2} \mathrm{sec}^{-1}$. $\mathrm{A} \mathrm{Nb}_{3} \mathrm{Sn}$ dipole has been designed with sufficient aperture for the separated beams needed for high-luminosity $\bar{p} p$ colliding beams. Indeed, the LBL group has just successfully tested a model block-coil dipole to 14.7 Tesla - a new world's record.

Several recent papers have calculated the reach of the Tripler for the Higgs boson [6] and for supersymmetry [7]. In both cases the physics reach of the Tripler is quite comparable to that of the LHC. Equally important, the initial-state processes by which new physics is accessed at the Tripler are quite distinct from those at LHC. At LHC, Higgs and SUSY are accessed primarily through gluon fusion and $\mathrm{W}$ fusion; at the Tripler, the valence antiquark content of the $\bar{p}$ gives comparable physics reach through $q \bar{q}$ annihilation channels. This complimentarity at the two facilities would be of great value in the challenging task of finding and studying new physics up to $\mathrm{TeV}$ mass

\footnotetext{
*Revised by Peter McIntyre, Texas A\&M University, June 17, 2001. This research funded by US Dept. of Energy, DE-FG03-95ER40924.

On leave from Lawrence Berkeley National Lab, Berkeley, CA 94720 USA.

${ }^{\dagger \dagger}$ p-mcintyre@physics.tamu.edu
}

scale. Indeed, phenomenology including the recent muon g-2 results indicates that the reach of the Tripler should extend over $\sim 85 \%$ of the SUGRA-compatible range of SUSY parameters [8].

\section{ARC MAGNETS}

The Tripler requires a ring of dipoles with $13 \mathrm{~T}$ field strength and quadrupoles with $250 \mathrm{~T} / \mathrm{m}$ gradient. The aperture must be $\sim 6 \mathrm{~cm}$, so that the beams of protons and antiprotons can be separated using electrostatic septa, except where they cross at the collision intersects. Figure 1 shows a cross-section of a quadrant of a block-coil dipole, which is adapted from our design for VLHC [5]. Its short-sample field is 13.4 Tesla $(4.2 \mathrm{~K})$, with a $6 \mathrm{~cm}$ aperture. The coil is excited with a single current. The dipole produces excellent field quality $\left(\mathrm{b}_{\mathrm{n}}<10^{-4} \mathrm{~cm}^{-\mathrm{n}}\right)$. A horizontal steel flux plate between the upper and central windings ( see Figure 1) provides strong suppression of multipoles from persistent-current magnetization currents.

The block-coil structure incorporates stress management [9], in which a support matrix of Inconel and titanium ribs and plates is integrated into the coil so that Lorentz stress is intercepted midway through the coil and bypassed. In this way the peak stress in the coil should never exceed $100 \mathrm{MPa}$, and the magnets should be robust against training. Preload is delivered using a set of expansion bladders that are filled with low-melt Wood's metal, and maintained under hydraulic pressure while the metal is cooled to solid.

The dipole also uses conductor optimization [9] in which the cables of the outer windings are wound from a combination of superconducting strands $\left(60 \% \mathrm{Nb}_{3} \mathrm{Sn}, 40 \%\right.$ $\mathrm{Cu})$ and pure $\mathrm{Cu}$ strands. In this way we provide the copper necessary for quench protection without the expense of drawing it into the superconducting strands.

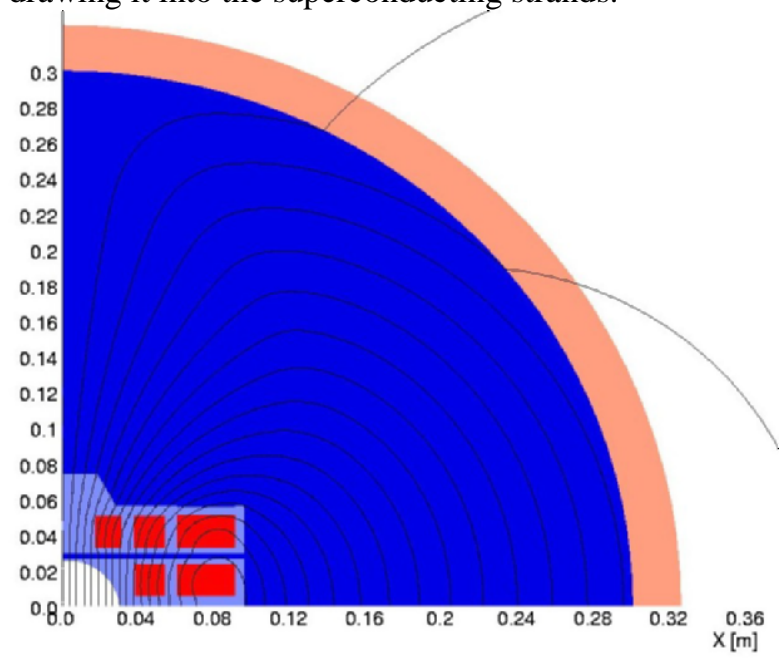

Figure 1. 13.3 Tesla $\mathrm{Nb}_{3} \mathrm{Sn}$ dipole for Tripler arcs. 


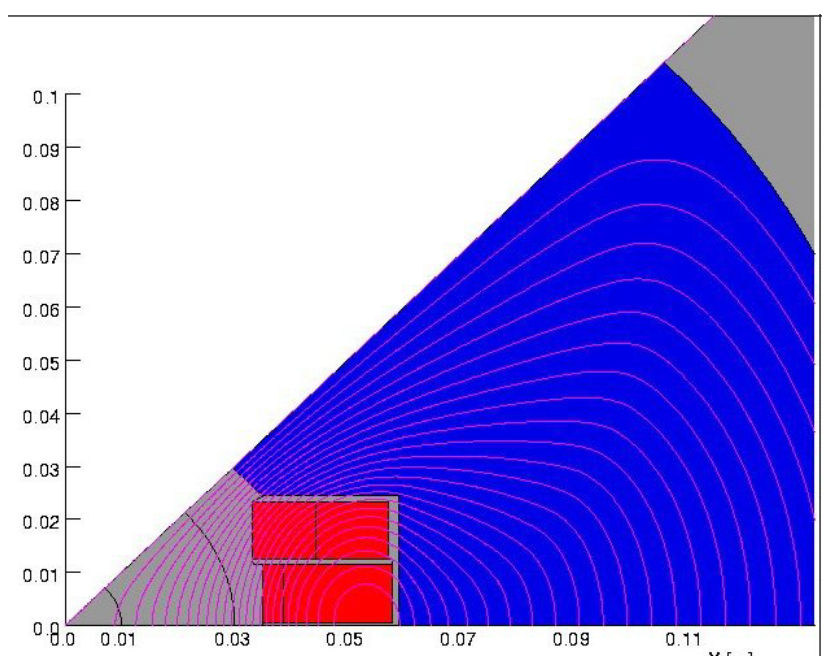

Figure 2. $290 \mathrm{~T} / \mathrm{m} \mathrm{Nb}_{3} \mathrm{Sn}$ quadrupole for Tripler arcs.

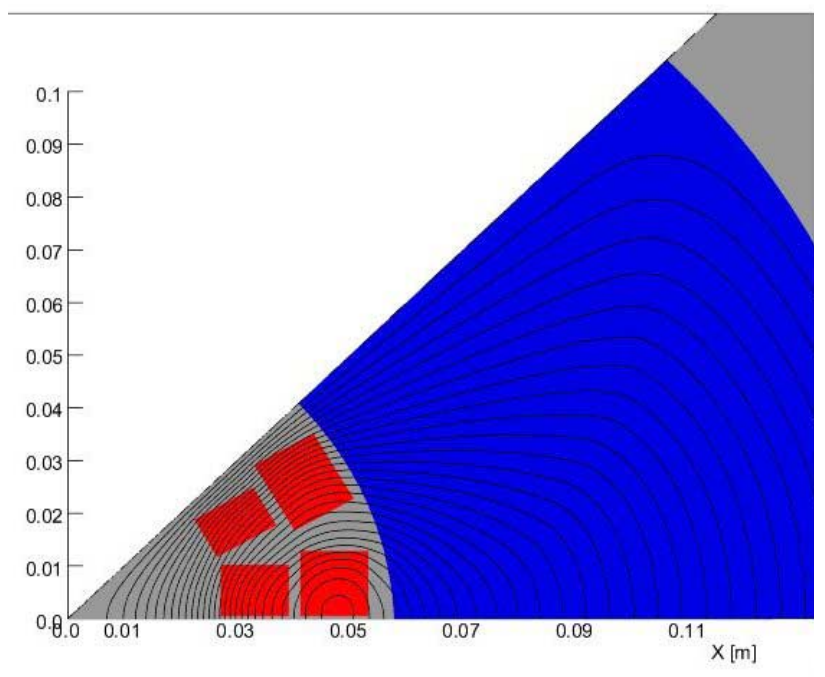

Figure 3. $415 \mathrm{~T} / \mathrm{m}$ quadrupole for Tripler low- $\beta$ insertion.

Table 1. Main parameters of arc dipoles and quadrupoles.

\begin{tabular}{l|cccl} 
& Arc & Arc & Low $\beta$ & \\
& dipole & quad & quad & \\
\hline Magnet length & 12 & 1.7 & $3.4,5.9$ & $\mathrm{~m}$ \\
Cold mass diam. & 64 & 35 & 40 & $\mathrm{~cm}$ \\
Short sample & $13.3 \mathrm{~T}$ & $255 \mathrm{~T} / \mathrm{m}$ & $415 \mathrm{~T} / \mathrm{m}$ &
\end{tabular}

field

Aperture diameter

Operating temp

Cables: \#strands,

strand diameter

\begin{tabular}{l|cccc} 
Inner windings & 36 & 21 & 21 & \\
& 0.93 & 0.92 & 1.02 & $\mathrm{~mm}$ \\
Outer windings & 50 & 26 & 34 & \\
& 0.68 & 0.75 & 0.65 & $\mathrm{~mm}$ \\
Coil @ 3 TeV: & & & & \\
Current & 17 & 12 & 20.5 & $\mathrm{kA}$ \\
$\quad$ Stored energy & 1.44 & 0.38 & $1.3,2.2$ & $\mathrm{MJ}$ \\
Inductance & 11 & 6 & $6.0,10$ & $\mathrm{mH}$
\end{tabular}
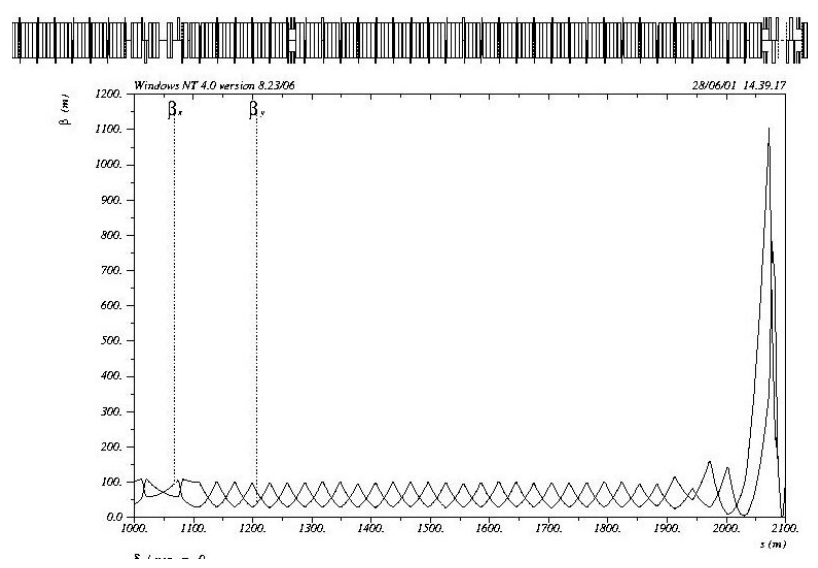

Figure 4. Lattice functions in Tevatron B-sector at $1 \mathrm{TeV}$.

We envisage fabricating the arc dipoles in lengths of 12 $\mathrm{m}$, twice that of the Tevatron dipoles. This would reduce by half the number of ends (where most installation and cost problems arise!) and speed the installation process.

Figure 2 shows a similar block-coil design for the arc quadrupoles. It uses the same techniques for stress management, preloading, and conductor optimization. It produces a short-sample gradient of $255 \mathrm{~T} / \mathrm{m}(4.2 \mathrm{~K})$ with an aperture of $6 \mathrm{~cm}$ diameter. The main parameters of the arc magnets are summarized in Table 1.

\section{LOW BETA INSERTIONS}

One of the most demanding technical elements in the Tevatron is the set of high-gradient quadrupoles that form the triplets at the low-beta insertions for the experiments. The Tevatron requires a quadrupole strength of $140 \mathrm{~T} / \mathrm{m}$ to produce $\beta^{*}=0.35 \mathrm{~m}$ at the collision point. Similar performance for the Tripler would require $420 \mathrm{~T} / \mathrm{m}$.

Because of the limited space in the Tevatron straight sections, the low- $\beta$ quadrupoles must be operated at the highest possible gradient in order to attain the design luminosity. Tripling the gradient of the low- $\beta$ quadrupoles of the Tevatron would require a gradient of $420 \mathrm{~T} / \mathrm{m}$. A design has been found which would attain $415 \mathrm{~T} / \mathrm{m}$ which required an aperture reduction to $5 \mathrm{~cm}$ and $1.8 \mathrm{~K}$ operating temperature. A continuing design study is in progress to ascertain if a more efficient design exists.

Table 2. Parameters of the Tevatron and the Tripler.

\begin{tabular}{l|lll} 
& Tevatron & Tripler & \\
\hline Collision energy & 1 & 3 & $\mathrm{TeV}$ \\
\# dipoles & 774 & 313 & \\
\# quads & 216 & 216 & \\
\# sectors & 6 & 6 &
\end{tabular}

Good field region

horizontal vertical

\pm 2
\pm 1.5
19.4
-22
35
140

$\pm 3$ $\pm 2.5$

$\mathrm{cm}$

Tune

19.4

Chromaticity

$\beta^{*}$ at collision pt.

$\bar{p}$ production rate 


\section{LATTICE}

The Tripler would operate with almost the same lattice as the Tevatron (Figure 4). The main properties of both lattices are summarized in Table 2.

The lattice for a sector arc of the Tevatron is

$$
\text { 2C,F,S,2B,D,4B, 12C }
$$

where $\mathrm{C}=\mathrm{F}, 4 \mathrm{~B}, \mathrm{D}, 4 \mathrm{~B}$ is a standard FODO cell

and $S$ is a $12.5 \mathrm{~m}$ warm straight section.

The $1 / 2$ cell length is $L_{1 / 2}=15 \mathrm{~m}$.

Each Tripler dipole has a length of $12 \mathrm{~m}$, twice the length of a Tevatron dipole, so each Tripler dipole replaces 2 adjacent Tevatron dipoles. We accommodate space for the additional separators by removing the first dipole on each side of each low- $\beta$ insertion. The loss of bending is compensated locally by installing special 14.5 $\mathrm{T}$ Tripler dipoles in the next 6 half-cells on each side of the insertion. The margin in field strength enables local correction of the orbit distortion produced by a missing dipole. The total sagitta of the local distortion is

$$
\delta=\left(3 L_{1 / 2}\right)^{2} \frac{e}{2 E}\left(B^{\prime}-B\right)=30 \mathrm{~cm}
$$

A total of 56 special dipoles is required for the two low$\beta$ insertions. A dipole design similar to Figure 1 but with thicker outer coil can provide the required additional field strength. The successful tests by the LBL group of a similar design to 14.7 Tesla demonstrate that this field strength is achievable.

Thus the lattice for a sector arc of the Tripler becomes

$$
\text { 2C,F,S,B,D,2B,9C,2C',F,2B', D, B', } \mathrm{S}^{\prime}
$$

where $\mathrm{C}=\mathrm{F}, 2 \mathrm{~B}, \mathrm{D}, 2 \mathrm{~B}$ is a standard FODO cell, $C^{\prime}=F, 2 B^{\prime}, D, 2 B^{\prime}$ is a cell with high-field bends,

and $S^{\prime}=6 \mathrm{~m}$ is the new warm straight section.

\section{RF, BEAM SEPARATION, AND ABORT}

The RF systems for the Tripler would be the same as those used for the Tevatron. The slower acceleration rate would not pose a problem for collider operation, and the bunch length at flat top would be about the same as that for the Tevatron.

The $\mathrm{p}$ and $\bar{p}$ bunches must be separated to prevent tune shift and associated beam growth from bunch-bunch interactions elsewhere than at the experimental insertions. The electric field in the Tevatron separators is about as large as is feasible, so that the aggregate length of separators must be tripled for the Tripler. The additional separators will be located in the new warm straight sections $S^{\prime}$ flanking each low- $\beta$ insertion.

Likewise the abort elements (kickers, septa, and dumps) must be lengthened to accommodate the higher beam energy. Preliminary analysis indicates that these provisions could be accommodated within available space in sector A.

\section{CONCLUSIONS}

The Tripler offers a possible upgrade to the Tevatron that would produce $\bar{p} p$ collisions with $6 \mathrm{TeV}$ center-ofmass energy and $10^{33} \mathrm{~cm}^{-2} \mathrm{~s}^{-1}$ luminosity. The upgrade requires only the replacement of the single magnet ring, in the same tunnel, with the same $\bar{p}$ source, the same injector, the same RF, and the same collider detectors. The inspiration in proposing the Tripler is to provide an upgraded physics program at Fermilab, with major discovery potential throughout the LHC era at a reasonable cost.

A more ambitious version of the same concept could be realized by building a new ring at Fermilab, still on the present site, with a circumference 2-3 times that of the Tevatron. This option would of course make possible correspondingly higher collision energy, and proper design of colliding beam insertions would yield higher luminosity. Luminosity would ultimately be limited by the $\bar{p}$ accumulation rate and by long-range tune shift.

\section{REFERENCES}

[1] H. Edwards, "The Tevatron Energy Doubler: a superconducting accelerator", Ann. Rev. Nucl. Part. Sci. 35, 605 (1985).

[2] E. Accomando et al., "The Tevatron Tripler: how to upgrade the Fermilab Tevatron for the Higgs boson and supersymmetry"

http://hepr8.physics.tamu.edu/hep/Tripler/tripler99.pdf

[3] R. Benjegerdes et al., "Fabrication and test results of a high field, $\mathrm{Nb}_{3} \mathrm{Sn}$ superconducting racetrack dipole magnet", paper TOAB009, these proceedings.

[4] A. Zlobin et al., "Development of $\cos \theta \mathrm{Nb}_{3}$ Sn dipole magnets for VLHC", paper RPPH085, these proceedings.

[5] R. Blackburn et al., "12 Tesla hybrid block-coil dipole for future hadron colliders". Proc. Applied Superconductivity Conf., Virginia Beach, VA Sept. 17-22, 2000.

[6] V. Barger et al., "Physics potential of a Tevatron Tripler", Phys. Lett. B478, 224 (2000).

[7] V. Krutelyov et al., "Prospect for searches for gluinos and squarks at a Tevatron Tripler", Phys. Lett. B505, 161 (2001).

[8] R. Arnowitt et al., "Muon g-2, dark matter detection, and accelerator physics", Phys Lett. B505, 177 (2001).

[9] C. Battle et al., "Optimized block-coil dipoles for future hadron colliders", Proc. Int'l. Conf. on Magnet Technology, Jacksonville, FL, Sept. 26-30, 1999. 